\title{
OPEN Inhibiting the two-component system GraXRS with verteporfin to combat Staphylococcus aureus infections
}

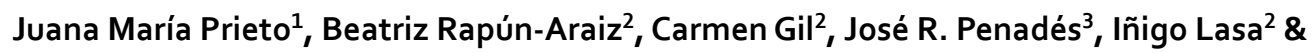 \\ Cristina Latasa ${ }^{1 凶}$
}

Infections caused by Staphylococcus aureus pose a serious and sometimes fatal health issue. With the aim of exploring a novel therapeutic approach, we chose GraXRS, a Two-Component System (TCS) that determines bacterial resilience against host innate immune barriers, as an alternative target to disarm S. aureus. Following a drug repurposing methodology, and taking advantage of a singular staphylococcal strain that lacks the whole TCS machinery but the target one, we screened 1.280 offpatent FDA-approved drug for GraXRS inhibition. Reinforcing the connection between this signaling pathway and redox sensing, we found that antioxidant and redox-active molecules were capable of reducing the expression of the GraXRS regulon. Among all the compounds, verteporfin (VER) was really efficient in enhancing PMN-mediated bacterial killing, while topical administration of such drug in a murine model of surgical wound infection significantly reduced the bacterial load. Experiments relying on the chemical mimicry existing between VER and heme group suggest that redox active residue C227 of GraS participates in the inhibition exerted by this FDA-approved drug. Based on these results, we propose VER as a promising candidate for sensitizing S. aureus that could be helpful to combat persistent or antibiotic-resistant infections.

Though the undeniable efficiency of anti-infective measures like vaccines and antimicrobials have made us believe that infectious diseases are nowadays under control, nothing could be further from the truth. An increasing number of studies alert that unless actions are taken, infections caused by antibiotic-resistant bacteria will kill an extra 10 million people a year worldwide by $2050^{1}$.

Staphylococcus aureus is one of the bacterial species whose manage is especially challenging due to the emergence of methicillin-resistant (MRSA) strains. With a population-weighted mean of invasive MRSA strains of about $17 \%$ in terms of European prevalence ${ }^{2}$, the major health care concern related to MRSA incidence lies in the limitations of currently approved treatments, which, in turn, leads to high rates of morbidity and mortality even in industrialized nations.

Despite of being an inoffensive colonizer of the nasal epithelium of one-third of the general population ${ }^{3}$, Staphylococcus aureus might become a dangerous life-threatening pathogen when it defeats host immune system, crosses the epithelial barrier and get access to deeper tissues like blood, dermis, gastrointestinal tract, heart valves or bones ${ }^{3,4}$. This biological versatility is based on a highly orchestrated regulation of circuits that sense a plethora of environmental signals and modulate gene expression for fine tuning crucial traits like cell-wall structure, biofilm formation or resistance to antibiotics. The core feature of such circuits is the two-componentsignaling transduction system (TCS) ${ }^{5}$, which is actually one of the most conserved and effective mechanisms in nature for coupling external stimuli and gene expression. In its most basic form, a canonical TCS normally consists of a membrane-bound histidine kinase and a cytosolic response regulator that, once phosphorylated, elicit appropriate changes in the cell by regulating gene expression, protein interactions, or enzymatic activity ${ }^{6}$.

Over the last decade, the scientific community has gained in-depth knowledge of the genes affected by specific staphylococcal TCSs, giving rise to a vast body of bibliography and information about mutants in the respective sensor kinases, response regulators and auxiliary genes. Thus, the pivotal role of AgrCA and SaeRS TCSs on

${ }^{1}$ RECOMBINA SL, Calle Nueva, 8 local 10, Mutilva 31192, Navarra, Spain. 'Laboratory of Microbial Pathogenesis, Navarrabiomed, Complejo Hospitalario de Navarra (CHN), Universidad Pública de Navarra (UPNA), IDISNA, 31008 Pamplona, Spain. ${ }^{3}$ Institute of Infection, Immunity and Inflammation, College of Medical, Veterinary and Life Sciences, University of Glasgow, Glasgow G12 8TA, UK. ${ }^{\star}$ email: cristina.latasa@gmail.com 
virulence gene expression or the involvement of BraRS and GraXRS in antibiotic resistance has been studied in great detail ${ }^{7-10}$. Furthermore, since TCSs are a matter of life and death to bacteria and they are not present in host's cells, these regulatory pathways have always been listed as promising antibacterial targets. Precisely based on the assumption that solution to present-day therapeutic limitations might somehow lie on impairing the way Staphylococcus senses and integrates environmental stimuli, significant effort in the form of ambitious High Throughput Screenings (HTSs) and Structure-Based Virtual Screenings (SBVSs) has been done to find new molecules with inhibitory effects on staphylococcal sensor kinases ${ }^{11}$. However, with few exceptions such as the molecule named walkmycin $\mathrm{B}^{12}$, biochemical screens normally identify a high number of compounds acting through nonspecific inhibitory mechanisms and thus render non-viable drugs in terms of clinical application ${ }^{11,13,14}$.

With the aim of designing a whole-cell drug discovery tool that could complement in silico docking and crystallographic analysis of kinase-ligands structure in TCS-targeting approaches, we decided to explore the potential of a recently developed staphylococcal strain that lacks the whole non-essential TCS machinery $(\Delta \mathrm{XV}$ strain $)^{15}$. Among all the TCSs whose individual contribution to a specific $S$. aureus phenotype has been defined, and based on its overall responsibility for resistance to host defenses like polymorphonuclear cells (PMNs) or cationic antimicrobial peptides, we selected GraXRS as a candidate of therapeutic target ${ }^{9,16-19}$. Additional evidence supporting our choice was given by a recent work showing that $S$. aureus uses this regulatory system to sense and adapt to the acidified phagolysosome in macrophages ${ }^{20}$, but also by several previous studies unveiling the potential of GraXRS to impact the bacterial capacity to colonize and survive on aortic valves in a rabbit endocarditis infection model ${ }^{18}$ or to play a crucial function in a murine model of systemic infection ${ }^{16}$. Most of these preceding articles conclude that mechanisms underlying GraXRS activity are related with changes in bacterial surface charge via its target downstream gene $m p r F$ and the operon dltABCD $9,17,18,21$.

In this study, we have used the $S$. aureus strain deprived of fifteen TCS $^{15}$ and isogenic derivatives containing exclusively the GraXRS TCS as a whole-cell platform to identify drugs that specifically target this signaling pathway. Upon evaluating the GraXRS-blocking activity of 1280 FDA-approved off-patent drugs, we found that molecules with antioxidant activity as acetylsalicylic acid, ascorbic acid, the porphyrin derivative verteporfin, or the flavonoid hesperidin, are capable of inhibiting the activity of GraXRS-dependent promoters. Among all the compounds, only verteporfin made a significant contribution to the susceptibility of $S$. aureus to human PMNs-mediated killing and rendered lower levels of bacterial colonization when its effect was assessed using an in vivo murine model. Though further analysis is needed to fully understand the precise molecular targets of verteporfin, data presented in this work suggest that the redox-active cysteine of GraS is required for this molecule to exert its inhibiting effect. Altogether, our results enlighten the potential of verteporfin as a supplement and(or) alternative antimicrobial therapy and provide evidence that this compound could be included in a recently described category of drugs known as "Potential Drugs for Repurposing against Infectious Agents".

\section{Results}

Design and validation of a GraXRS-focused screening platform. With the aim of designing a highly specific GraXRS-targeting screening assay, we first restored the GraXRS TCS into the chromosome of $S$. aureus $\triangle \mathrm{XV}$ strain, which only contains the essential WalKS TCS system in its genome. The resulting $S$. aureus $\triangle X V$ Gra-RES strain, together with the corresponding $S$. aureus MW2 wild type strain and the single GraXRS mutant derivative, were transformed with two different reporter plasmids in which lacZ expression depends on the GraXRS-regulated promoters of $m p r F$ and $d l t \mathrm{X}^{9}$. As shown in Fig. 1, transcriptional activity of reporter genes was barely detectable in the GraXRS deficient strains (single and multiple $\Delta \mathrm{XV}$ mutants), whereas $m p r F$ and $d l t \mathrm{X}$-based reporter constructs were highly induced in the GraXRS containing strains (wild type and $\Delta \mathrm{XV}$ GraRES). As an additional test for evaluating the behavior of the reporter strain-set, positive response of the GraXRS-dependent promoters to the presence of sublethal concentrations of colistin was also analyzed. Noticeably, and confirming the concept of TCS as self-sufficient modules previously envisioned ${ }^{15}$, lacZ expression driven by $d l t \mathrm{X}$ promoter was essentially equal in the wild type and graXRS restored genetic backgrounds. This result validates both the use of the GraXRS restored strain and dltXP::lacZ transcriptional fusion for high-throughputscreening designs aimed at the discovery of GraXRS-blocking drugs and molecules.

Primary and secondary screenings. From the initial screening of the 1280 drugs included in the Prestwick library (https://www.prestwickchemical.com/libraries-screening-lib-pcl.html), we selected 77 compounds that led to reduced activity of $d l t X$ promoter by more than $55 \%$ as determined by beta-galactosidase activity (see table in the supplemental material). Because the intention of this work was to repurpose those FDA-approved drugs that specifically targeted GraXRS-mediated signaling pathway, and this TCS has been shown to be crucial for bacterial growth under acidic conditions ${ }^{15}$, the capacity of the selected compounds to affect $\mathrm{OD}_{600}$ values at pH 5.5 was tested, expecting a significant growth arrest in the presence of GraXRS-blocking compounds. Following a similar approach, the dose-response behavior of selected compounds was evaluated. To do so, both bacterial growth and $d$ ltx $P$ transcriptional activity were quantitatively assessed in the presence of variable concentrations, ranging from 0 to $20 \mu \mathrm{M}$, of selected drugs (Fig. 2C).

In order to reinforce the involvement of GraXRS TCS in the phenotypic outcomes rendered by the drugs to be chosen, their ability to down regulate the GraXRS-dependent alternative $m p r F$ promoter was considered as an additional selective criterion.

To verify the specificity of GraXRS for the selected compounds, we restored a different TCS, saeRS, into the $\triangle \mathrm{XV}$ genome. In this case, the reporter lacZ gene was transcriptionally fused to sec4 promoter, which has previously described as part of SaeRS regulon ${ }^{22}$. No shift in the transcription levels of sec4P::lacZ fusion appeared when $\triangle X V$ Sae-RES were incubated in the presence of each selected compound. 

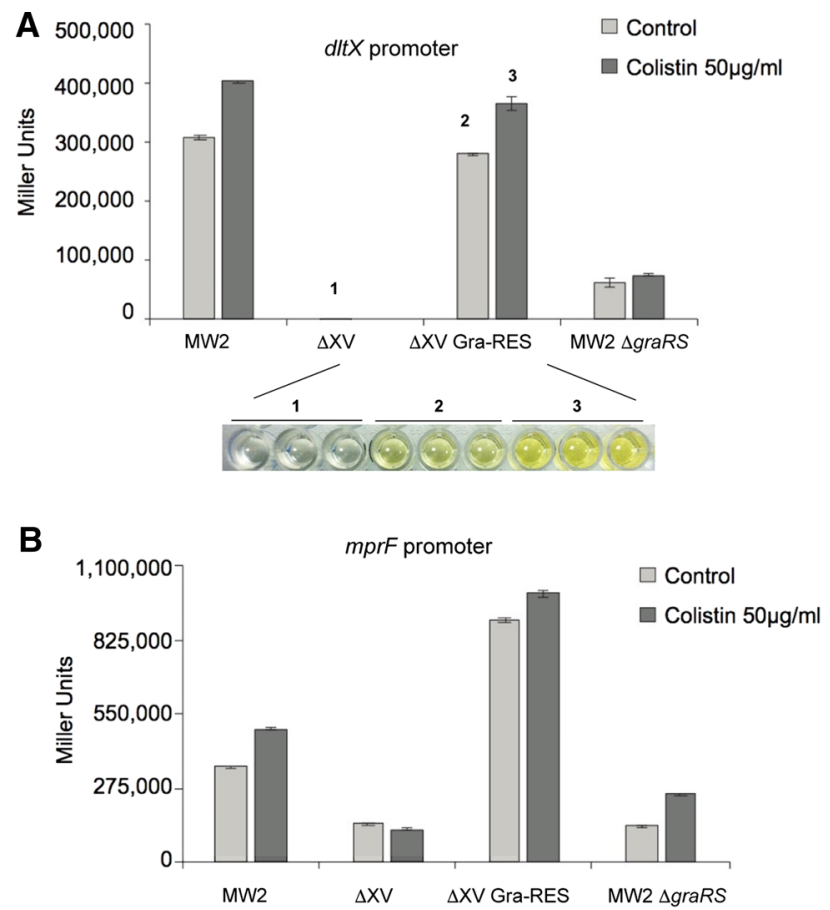

Figure 1. Validation of GraXRS-dependent transcriptional fusions. Graphic illustration of GraXRS-dependent activity of $d l t \mathrm{X}(\mathbf{A})$ and $m p r F(\mathbf{B})$ promoters in $S$. aureus MW2 wild type, $\Delta \mathrm{XV}, \Delta \mathrm{XV}$ Gra-RES and $\Delta g r a \mathrm{RS}$ strains. Transcriptional activity in TSB medium (light gray bars) and TSB supplemented with colistin $50 \mu \mathrm{g} / \mathrm{ml}$ (dark grey bars) is presented. Means and standard deviations values are shown from at least three independent experiments. A visual example showing different degrees of GraXRS activity [ $\triangle \mathrm{XV}(1), \Delta \mathrm{XV}$ Gra-RES (2) and $\triangle \mathrm{XV}$ Gra-RES in the presence of colistin (3)] in the 96-well format is also shown.

Finally, and though equivalency between wild type and GraXRS restored strains in terms of GraXRS-mediated sensing had been previously validated, the possibility that other TCSs or derived circuits absent on $\triangle \mathrm{XV}$ GraRES could somehow affect $d l t X$ transcriptional activity was considered. In order to address this issue, the MW2 wild type strain was transformed with the dltX-derived reporter plasmid and beta-galactosidase activity was measured in the absence and presence of mentioned compounds.

As shown in Fig. 2, the whole consecutive screening process significantly restricted the number of active compounds from almost 80 to 5. Acetylsalicylic acid (ASA), hesperidin (HES), ascorbic acid (VITC), verteporfin (VER) and troglitazone (TGZ) were capable of inhibiting bacterial growth under acidic conditions, affected $m p r F P$ activity negatively and exerted a suppressing effect on $d l t X P$ in the wild type genetic background. Among this final group of drugs, TGZ displayed an additional negative impact on SaeRS, and thus was firstly considered as a potential multi-target drug. However, since this molecule was withdrawn in 2000 due to high risk of hepatotoxicity, only ASA, HES, VITC and VER were contemplated as real candidates for therapeutic reposition. Curiously, though these four compounds show no common (known) pharmacological features, they are all chemically classified as redox-active drugs.

In vitro effect of selected compounds: phagocytosis and killing of $S$. aureus by human PMNs. Deepening into the anti-virulence potential of selected drugs, we next proceeded with an in vitro assay in which the bacterial susceptibility to phagocytosis and killing by human polymorphonuclear cells was assessed. Considering that GraXRS has been shown to have a pivotal role for S. aureus to resist PMN attack ${ }^{20}$, we isolated this cellular fraction from peripheral human blood and the effect of ASA, HES, VITC and VER on MW2 susceptibility to these immune cells was assessed. In all cases the process was boosted by opsonic antibodies naturally present in human sera due to unavoidable exposure to S. aureus. After incubating PMNs-S. aureus MW2 suspensions for $30 \mathrm{~min}$ in the absence or presence of the four selected compounds ( $5 \mu \mathrm{M}$ each), removal of extracellular bacteria via gentamicin exposure, and subsequent lysis of eukaryotic cells at basic $\mathrm{pH}$, bacterial viability was estimated via plate counting. Data shown in Fig. 3 prompted us to conclude that the presence of ASA, HES and VITC caused a slight increase in the sensitivity to PMN attack, while the effect of VER on reducing the number of surviving intracellular bacteria was substantially obvious and statistically significant (Fig. 3). As a result, from this in vitro approach, we chose VER as the sole candidate to proceed with the next assays.

Effect of verteporfin in a murine model of wound infection. Following on from the in vitro testing, we next evaluated VER using the murine model of wound (or surgical site) infection. In this in vivo approach, a silk suture contaminated with $S$. aureus MW2 or MW2 $\operatorname{graXRS}$ strain $\left(4.5 \times 10^{5} \mathrm{cfus} \mathrm{cm}^{-1}\right)$ was used for sewing 
A

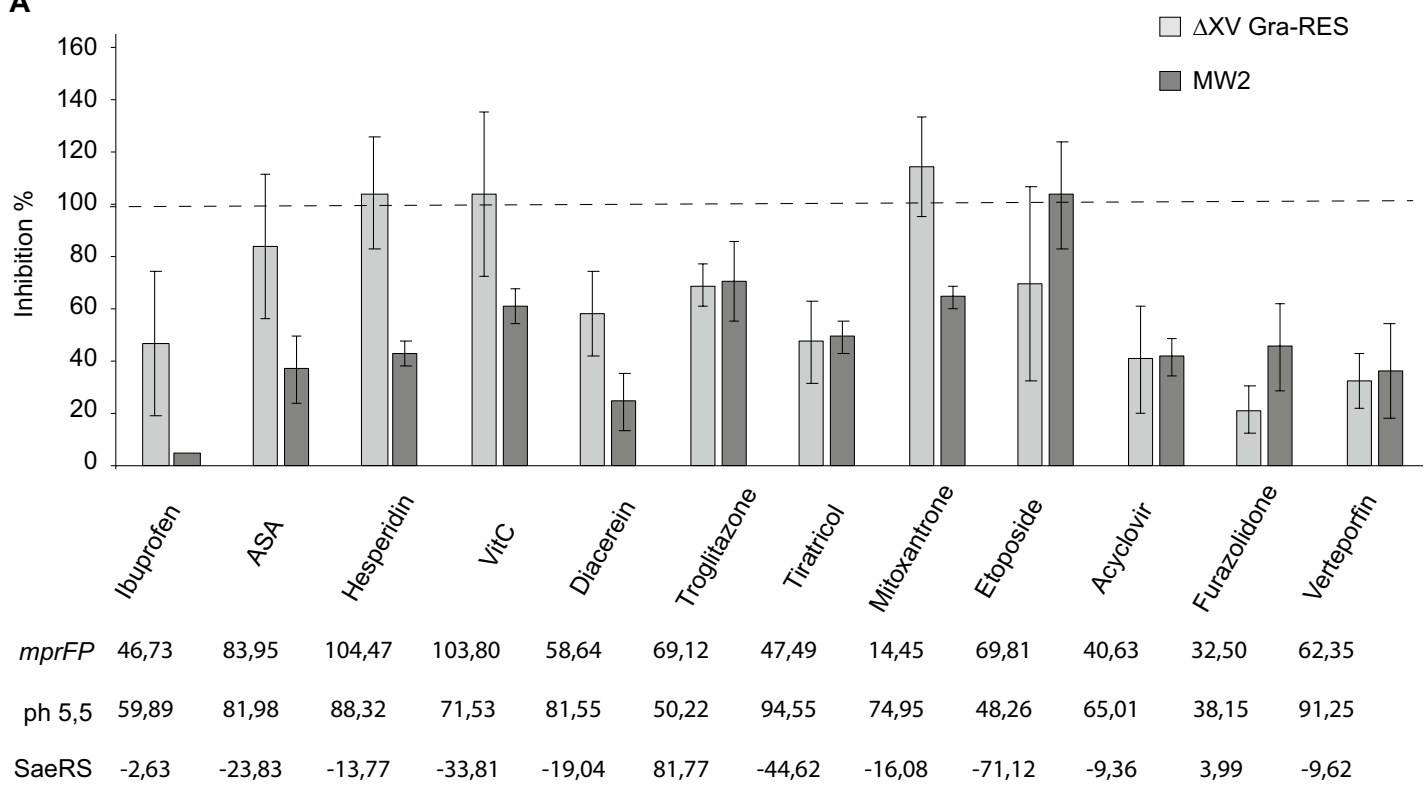

B

$\begin{array}{lllll}\text { ASA } & \text { Hesperidin (HES) } & \text { VitC } & \text { Diacerein (DIA) } & \text { Tiratricol (TIR) }\end{array}$

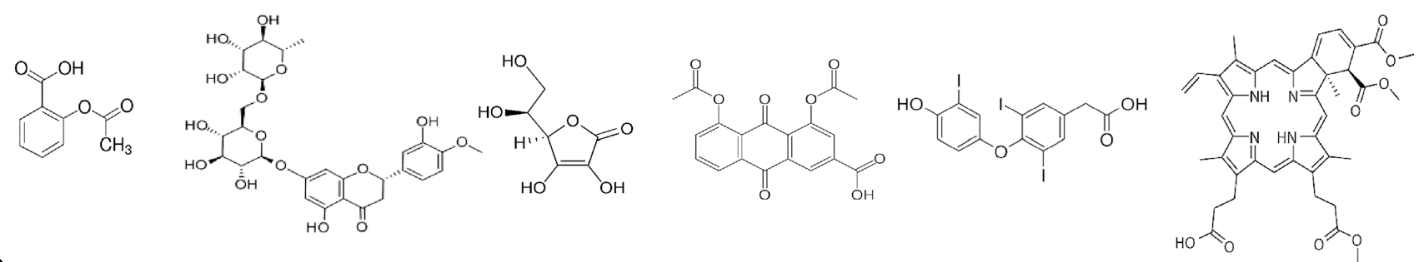

C
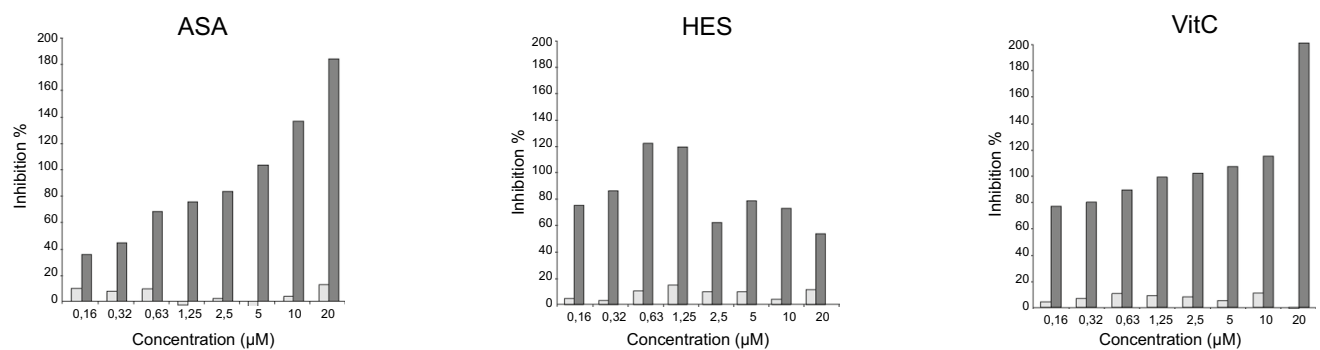

Gorwth Inhibition \%

dltX inhibition \%
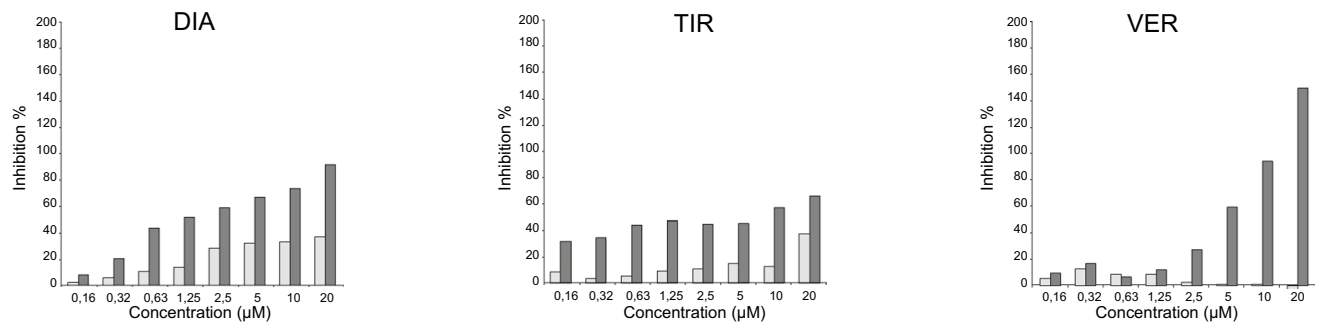

Figure 2. Screening results. (A) Graphic illustration of GraXRS-dependent activity of $d l t X$ promoter in the presence of selected drugs $(10 \mu \mathrm{M})$ in S. aureus $\triangle \mathrm{XV}$ Gra-RES (light gray bars) and MW2 wild type strains (dark grey bars). Data are presented as relative M.U. values obtained in the absence of any compound for $S$. aureus $\triangle \mathrm{XV}$ Gra-RES and MW2 wild type strains respectively. Values exceeding $100 \%$ correspond to those cases where the observed transcriptional activity is lower to that showed by $\Delta \mathrm{XV}$ and/or bacterial growth is inhibited. Standard deviations values correspond to at least three independent experiments. Results regarding additional selection criteria including the repression of the GraXRS-dependent alternative $m p r F$ promoter, significative inhibition of wild type strain under acidic growth conditions ( $\mathrm{pH}$ 5.5) and transcriptional co-inhibition of the SaeRS-dependent sec4 promoter are presented. (B) Structure of selected drugs included in drug bank profiles (https://www.drugbank.ca) is shown. (C) Graphic illustration of Dose Dependency profiles in terms of $d l x P$ inhibition exhibited by selected compounds. Data are presented as relative M.U. values obtained in the absence of any compound for S. aureus $\triangle \mathrm{XV}$ Gra-RES (dark grey bars). Relative percentage of survival in the presence of increasing doses of compounds is also included (light grey bars). 


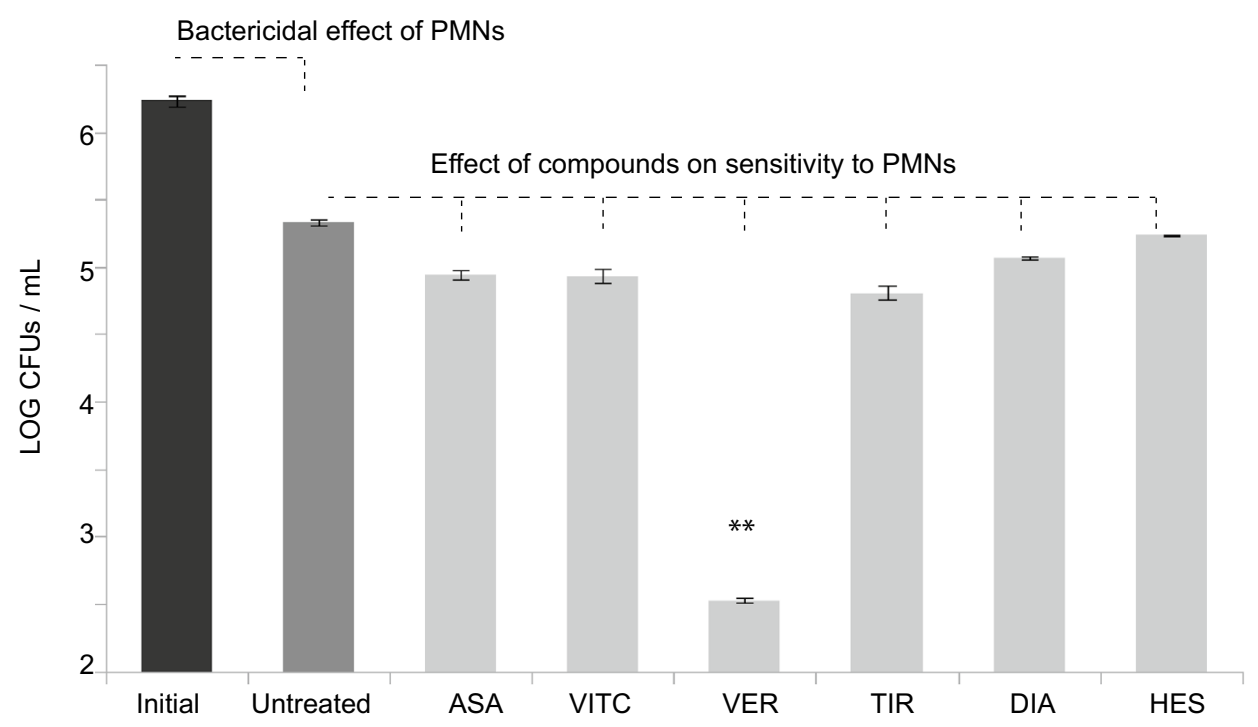

Figure 3. Effect of selected drugs in the susceptibility to phagocytosis and killing by human PMNs. Graphical scheme of bacterial counts expressed as log values after incubating PMNs and S. aureus MW2 wild type suspensions for $30 \mathrm{~min}$ in the presence or absence of four selected compounds ( $5 \mu \mathrm{M}$ each), subsequent gentamicin treatments and lysis of eukaryotic cells (see "Materials and methods" section). Means and standard deviations are presented, illustrating the bactericidal effect of PMNs and the additional contribution of ASA, VITC, VER, TIR, DIA and HES. In the case of VER, two asterisks denote an associated p value of 0.006 when ANOVA and Tukey's pairwise post hoc tests were applied according to prior normality and homoscedasticity tests.

up a previous incision on the back of the mice. VER was topically applied 2 and $24 \mathrm{~h}$ after suture implantation using hydrogel-based formulations that contained either no active ingredient or the porphyrin under study at a low $(0.125 \mathrm{mg} / \mathrm{kg})$ or a high $(2.5 \mathrm{mg} / \mathrm{kg})$ dose. Assessment of the infection was performed by counting viable bacteria in tissue homogenates that were obtained upon animal euthanasia, $24 \mathrm{~h}$ after the last treatment.

As shown in Fig. 4, the significantly lower quantity of viable bacteria present in tissue samples that had been infected with the GraXRS deficient strain unveiled the critical role of this TCS in surgical wound infections. When the effect of VER was assessed, data showed that topical administration of this drug significantly reduced the bacterial load in a dose-dependent manner. Noticeably, application of VER at a low dose led to a similar degree of bacterial colonization to that followed by the implantation of sutures that had been contaminated with the GraXRS lacking strain. While such a result reinforces the potential of VER as a GraXRS inhibitory drug, its specificity is suggested by the fact that the observed outcome after treatment of wounds infected with the wild type strain is certainly evident but it becomes almost imperceptible in the case of $\Delta g r a X R S$-associated infection. Equally certain, however, is that high doses of VER could exert a GraXRS-independent and/or antibiotic effect.

Exploring the mechanistic basis of verteporfin. Because VER is a tetradentate chelating porphyrin ${ }^{23}$ that might be involved in redox sensing, just like heme complex, and such molecules are normally sensed through thiol-based switches, we also analyzed the contribution of the single redox-active cysteine present in GraS, C227, to VER effect ${ }^{24}$. To do so, C227 was mutated to S or A in $\triangle X V$ Gra-RES background and transcriptional activity of $d l t X P$ in the resulting $\triangle X V$ Gra-RES S(C227-S) and $\triangle X V$ Gra-RES S(C227-A) strains was measured. An additional strain in which GraS H129 amino acid, the residue that undergoes phosphorylation upon activation of the kinase, had been mutated to $\mathrm{Q}$ was also constructed and included as a reference of complete GraXRS inactivation. As shown in Fig. 5A, data verified that replacement of cysteine by another residue had a negative impact on GraXRS activity, being such an outcome dependent on the polarity of the substituted amino acid. Thus, C227-A (non-polar) GraS isoform led to a lower degree of transcriptional activity of dltXP in comparison to that showed by the isoform in which $\mathrm{C} 227$ had been mutated to the polar amino acid serine. In accordance with this observation, bacterial growth arrest under acidic conditions, a phenotype that strictly reflects GraXRS status, also showed dependence on the mutation polarity (Fig. 5B). When the effect of VER was assessed, transcriptional data revealed that GraXRS repression led by mutations was far from being as drastic as the one achieved by VER (Fig. 5A), discarding the possibility of considering the $\mathrm{C} 227$ redox switch as the exclusive mechanism underlying VER effect. However, impairment of the signaling via C227 resulted in the insensitivity to VER, fact that suggests that intermolecular cysteine-disulfide-bond formation is required, though not entirely, for VER to have a blocking GraXRS-dependent effect. 


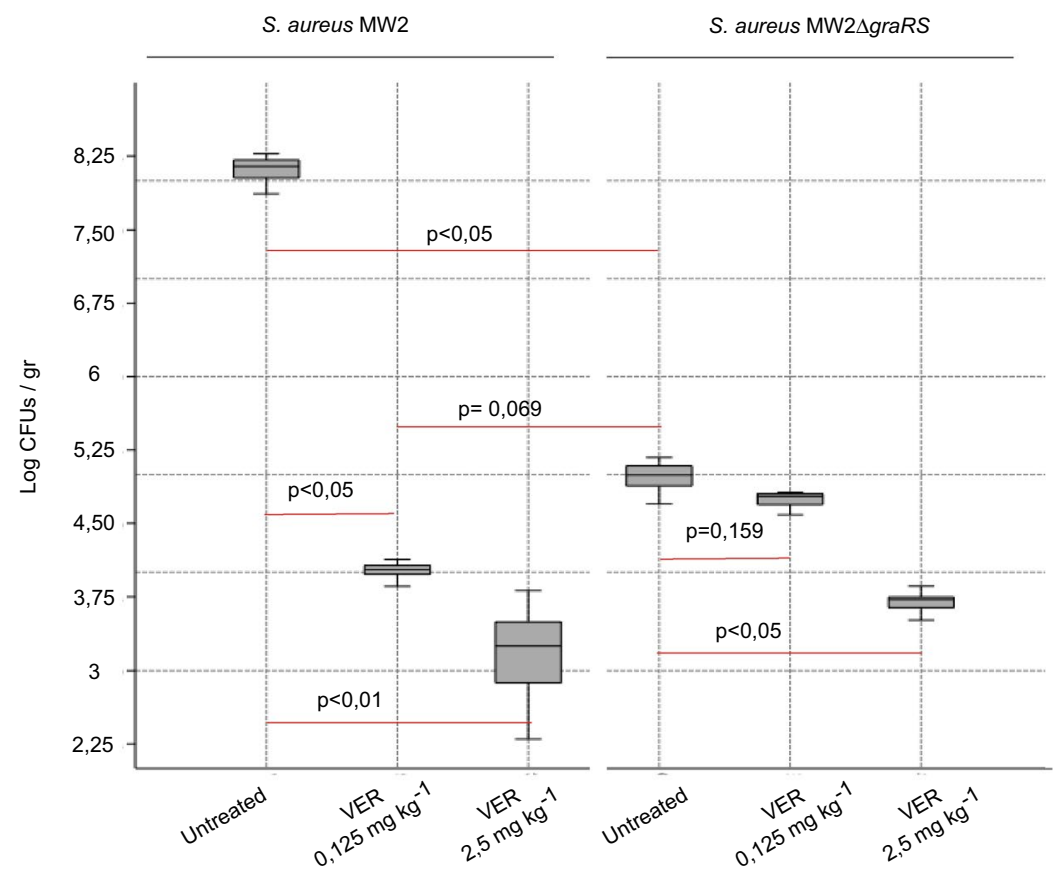

Figure 4. Effect of VER in a murine model of surgical wound infection. BOX-PLOT illustration (PAST statistical package) of bacterial counts expressed as log values per gram of wound tissue homogenates. Different treatments include the topical administration of hydrogel-based formulations with two doses ( 0.125 and $2.5 \mathrm{mg} \mathrm{kg}^{-1}$ ) of VER, 2 and $24 \mathrm{~h}$ after making an incision that was immediately infected with a surgical suture contaminated with $4 \times 10^{5}$ CFUs of $S$. aureus MW2 wild type or $\triangle$ graXRS strain. Hydrogel without any active pharmaceutical ingredient was applied to control mice. All the animals were euthanized $24 \mathrm{~h}$ after the last treatment. Apart from visual interpretation in accordance to IQR overlapping, data were statistically analyzed via Kruskal-Wallis and post hoc Mann-Whitney test. Corrected p-values corresponding to relevant post hoc statistical comparisons are also shown.

A

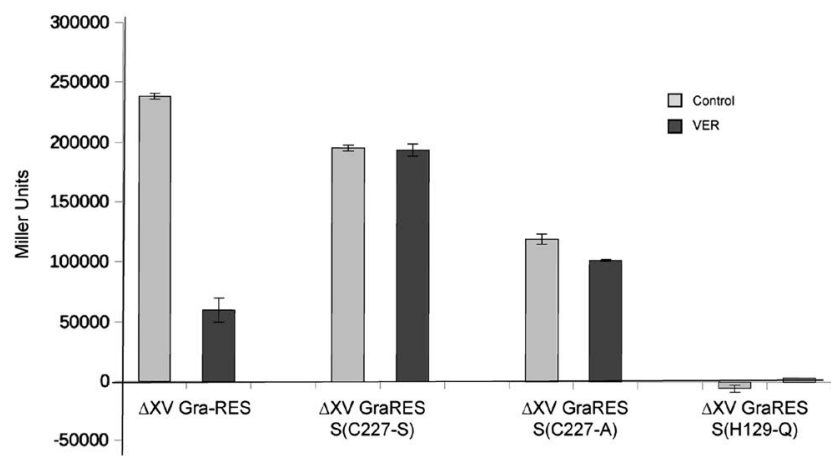

B

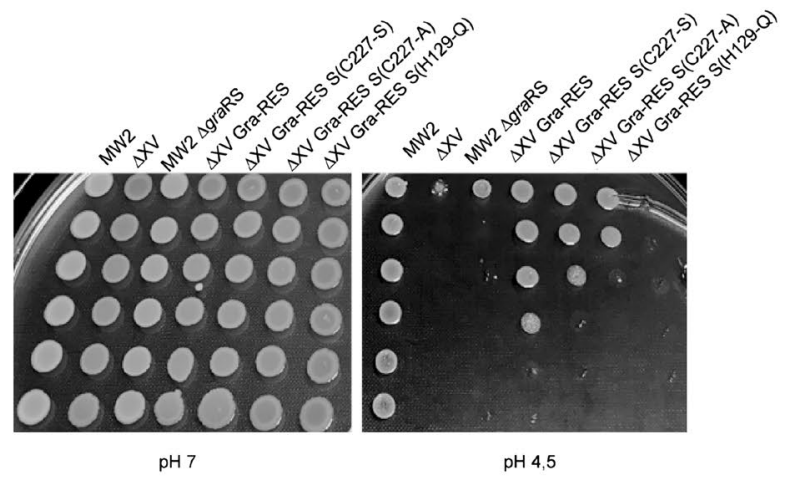

Figure 5. C227-mediated redox switch involvement in the sensitizing effect exerted by VER. (A) Transcriptional activity of GraXRS-dependent dltX promoter in S. aureus $\Delta$ XV Gra-RES, $\triangle X V$ Gra-RES S(C227-S), $\triangle$ XV Gra-RES S(C227-A) and $\triangle$ XV Gra-RES S(H129-Q) strains is illustrated both in the presence (dark grey bars) or absence (light gray bars) of VER $(10 \mu \mathrm{M})$. Means and standard deviations values are shown form at least three independent experiments. (B) Pictures illustrating the capacity of serially-diluted suspensions of bacterial strains $S$. aureus MW2 wild type, $\Delta \mathrm{XV}$, $\Delta$ graRS, $\Delta \mathrm{XV}$ Gra-RES, $\Delta \mathrm{XV}$ Gra-RES S(C227-S), $\Delta \mathrm{XV}$ GraRES S(C227-A) and $\triangle$ XV Gra-RES S(H129-Q) to grow in neutral (left) and acidified (right) TSA medium are also included in this figure. 


\section{Discussion}

Current strategies aimed at antimicrobial discovery prioritize innovative concepts like alternative molecular mechanisms of action, new natural product sources, pro-drugs, or even approved compounds that were originally intended for other therapeutic indications, as it is the case of the present work (Statement of Antimicrobial drug discovery, EASAC-2014 ${ }^{25}$ ). Particularly speaking of S. aureus, and discarding the essential TCS WalKR/S as the antibiotic target per excelence, continuous efforts are being made toward the discovery of inhibitors of TCS involved in virulence and biofilm formation (for review ${ }^{26-28}$ ). Impairment of these non-essential biological pathways has the advantage of requiring a lower plasmatic dose compared with a MIC, reducing the tendency to resistance and minimizing side effects on neutral and beneficial microbiota that colonize treated human or animal hosts ${ }^{29}$. Up to date, several novel and previously approved drugs with the capacity to exert an inhibitory effect on Agr, SaeRS, and ArlRS TCSs have been described (for review ${ }^{26-28}$ ) but, to our knowledge, GraXRS had never been considered as a target for disarming S. aureus. The involvement of this TCS in the resistance to antimicrobial peptides and macrophages had already been envisioned in several occasions $s^{9,30,31}$, but it has just been recently proven that GraXRS is entirely responsible for the response to $\mathrm{pH}$ inside acidified macrophage phagolysosomes ${ }^{15,20}$. These premises led us to consider GraXRS as a clear target to counteract $S$. aureus response to innate host immunity and impede replication of the pathogen in the acute stage of systemic infection.

Taking advantage of the $S$. aureus strain that lacks its complete sensorial TCS network ${ }^{15}$, we developed a series of reporter strains that could be helpful for selecting compounds capable of blocking transcriptional activity of the GraXRS-dependent $d l t X$ promoter. Working with $\triangle X V$ Gra-RES strain gave us the opportunity to perform a bioassay where bacterial sensing entirely depends on GraXRS (and WalkRS), thus reducing the probability of selecting off-target drug candidates. Proof of this last claim is the fact that among selected drugs, only one of them (Troglitazone) displayed a GraXRS-SaeRS multi target effect.

At the time of deciding the type of compound to be tested for the identification of GraXRS inhibitors, we, as many other researchers, opted for the drug-repurposing approach. This strategy is based on the identification of "off" antimicrobial targets for drugs that were approved for other clinical diseases ${ }^{29}$, hence bypassing the financial and regulatory barriers that have to be overcome to bring a drug to market. At present day, this concept of repurposing has gained renewed interest and a novel category of drugs known as "Potential Drugs for Repurposing against Infectious Agents" is exponentially thriving ${ }^{32}$ By way of example, the old antimalarial drug chloroquine is being tested as SARS-CoV-2 inhibitor ${ }^{33}$. Though the precise mechanistic basis of their effect remains to be completely elucidated, current candidate PDRIAs targeting S. aureus SaeRS and/or AgrTCSs are floxuridine, streptozotocin and diflunisal ${ }^{28,34,35}$.

The screening methodology applied here consisted in the analysis of changes in dltXP transcriptional activity, followed by several consecutive steps where additional criteria like the effect on an alternative GraXRS-dependent promoter, TCS-selectivity, or the determination of bacterial growth in the presence of selected drugs under acidic conditions were applied. The overall process ended up in the selection of five candidate drugs: acetylsalicylic acid (ASA), hesperidin (HES), ascorbic acid (VITC), verteporfin (VER) and troglitazone (TGZ). Curiously, all compounds are classified as redox-active drugs. While ASA, HES, VITC and TGZ are commonly sorted as antioxidant molecules, VER can induce oxidative stress through the production of free radicals or be alternatively combined with soluble metals and display a redox potential similar to that showed by the heme complex ${ }^{23}$. These observations are in agreement with the previously unveiled connection between GraXRS and oxidative stress, evidenced by the deciphering of the GraXRS regulon and the proved essentiality of this TCS in staphylococcal resistance to redox compounds like paraquat or $\mathrm{H}_{2} \mathrm{O}_{2}{ }^{9}$. Furthermore, a recent RNA-seq transcriptomic approach has just corroborated the involvement of GraXRS, collectively with VraSR, SaeRS, MgrA, SigB or Fur, in the cell response to thiol-oxidative stress ${ }^{36,37}$.

After proving that VER was the only compound really capable of sensitizing bacteria against the effect of human PMNs, this compound was further examined using a murine model of surgical wound infection. This model has been previously used to assess the effect of systemic and topical antimicrobial agents, finding a close correlation with efficacy in clinical trials with human subjects. Noticeably, our results provided further evidence concerning the critical role of GraXRS in skin and wound infections and insinuated a pharmaceutical potential of VER as a novel local treatment for S. aureus infections. Since bacterial count after the infection with the wild type strain and subsequent treatment with $0.125 \mathrm{mg} \mathrm{kg}^{-1}$ of VER was quite similar to that proceeding from the infection with a GraXRS negative strain, the effect exerted by the porphyrin derivative seems to be highly dependent on the activity of this TCS.

When the possible path(s) of how VER inhibits the activity of GraXRS was envisaged, we thought of VER as a heme-like porphyrin capable of binding iron in different oxidation states ${ }^{23}$. Recent transcriptomic studies conducted with the constitutively-active forms of staphylococcal kinases have unveiled the involvement of GraXRS in the regulation of heme synthase A (MW_RS05355; ${ }^{38}$ ), fact that led us to attach importance to the chemical mimicry between both molecules. Since porphyrins are normally involved in thiol-based molecular switches ${ }^{24}$, the unique potentially redox-active residue in GraS, C227, was considered a potential molecular target of VER. To address this question, the impact of C227 mutations to S and A on GraXRS activity and VER sensitivity was assessed. To our knowledge, the results achieved in this work by punctual mutations of C227 have evidenced for the first time that this cytosol-located redox-active residue actually participates in GraS kinase activity. This line of reasoning, which has support from studies that have characterized other bacterial kinases like $\mathrm{AcrB}^{39}$, showed that GraS degree of silencing depended on the polar nature of the amino acid that substituted C227 and suggested the involvement of this redox-switch as a potential molecular target concerning VER effect. However, and though C227 substitutions led to insensitivity to VER, the inhibitory outcome yielded by mutations was not as drastic as the one achieved by exposure to VER, suggesting that additional molecular paths must be involved in this process. An additional candidate that might be considered is Stk1, the unique serine-threonine kinase 


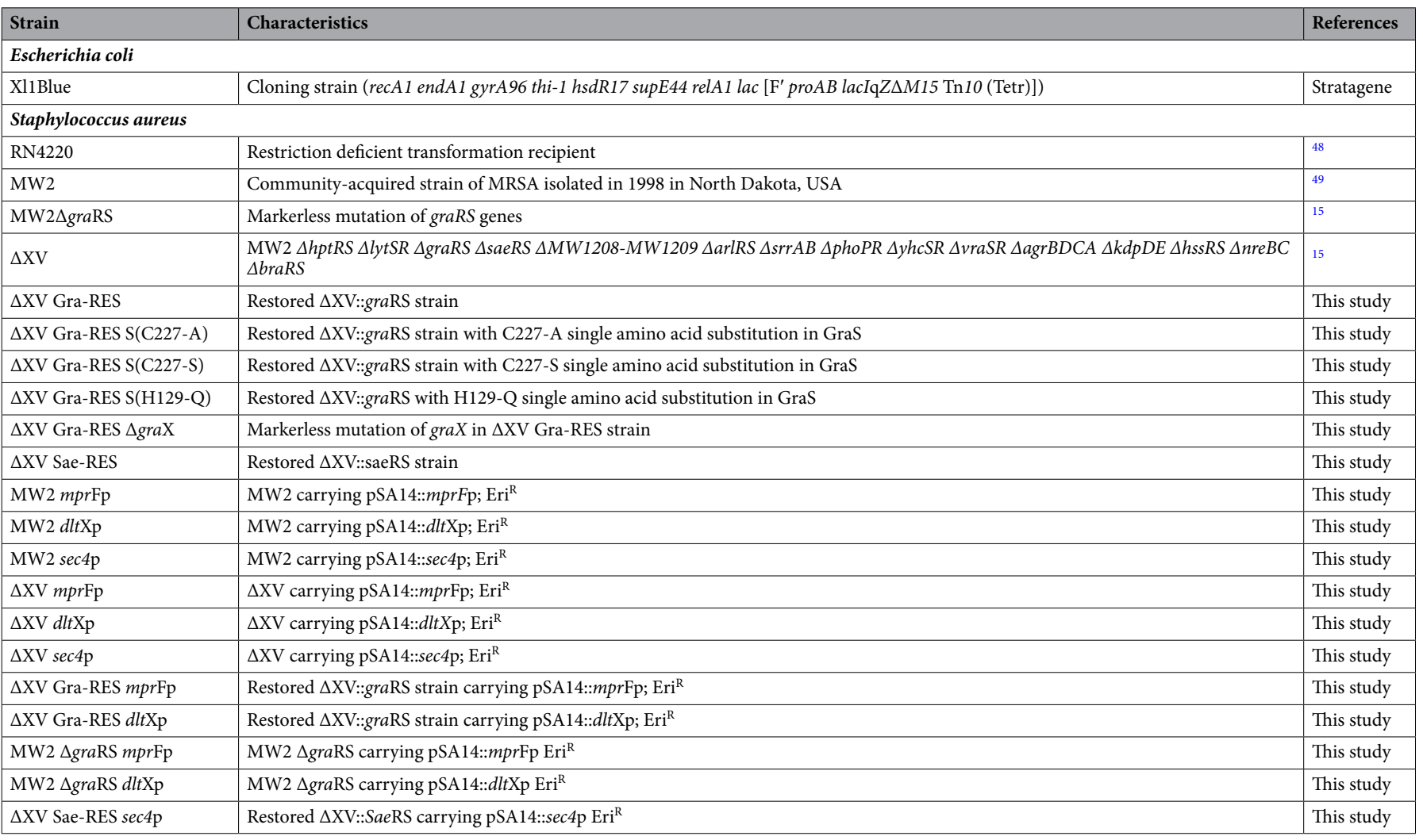

Table 1. Strains used in this study.

which cross-phosphorylates $\mathrm{GraR}^{40}$ and shows homology with OXR1, one of the recently discovered mammalian target of VER that, curiously, is also related to oxidative stress ${ }^{41}$. However, in accordance with Fridman et al. ${ }^{40}$, we have verified that both Stk1 and GraS-mediated phosphorylation on GraR as T128, T130, T149 and D51 respectively are equally required for full $d l t X$ expression (data not shown), fact that seriously complicates the use of our reporter systems when it comes to holding Stk1 accountable for intervening on VER effect.

Could VER be considered a viable antimicrobial candidate? Though we are fully aware that further in vitro and in silico studies helping to understand the whole molecular scenario underlaying VER effect and alternative in vivo approaches or definition of strategic dosages are some pending issues to claim a novel anti-virulence pharmaceutical indication for VER, we are convinced that this drug presents some strengths, apart from those inherent to anti-virulence drugs, that might be worth considering. In terms of pharmacology, for instance, VER excretion is dependent on hepatic function, while many antibiotics primarily undergo elimination via kidney filtration. Thus, VER could be administered to patients suffering from kidney disease, or to those who were being concomitantly treated with antibiotics that are prone to cause nephrotoxicity (e.g. vancomycin $)^{42}$. On the other hand, though side effects associated to parenteral administration of VER include hypersensitivity reactions or blood pressure alteration, these symptoms have lower mean severity ratings in comparison to those showed by many antibiotics (ema.europa.eu/Find medicine/Human medicines/European public assessment reports). Finally, taking into account that we have also observed that VER-containing topical formulations are effective and that further studies might confirm that chemical mimicry between porphyrin derivatives and heme group could actually be harnessed for disarming $S$. aureus, our results may be considered as a step forward in re-proposing VER as a plausible alternative in combating antimicrobial resistance.

\section{Materials and methods}

Bacterial strains, plasmids, oligonucleotides and culture media. Bacterial strains, plasmids and oligonucleotides (purchased to IDT) are listed in Tables 1, 2 and 3 respectively. Escherichia coli XL1blue strain was grown in LB broth and LB agar. Staphylococcus aureus strains were grown on trypticase soy broth (TSB), trypticase soy agar (TSA), trypticase soy broth with $0.2 \%$ of glucose (TSB-glu), Mueller Hinton (MH) and $\mathrm{B} 2$ medium [ $1 \%$ casein hydrolysate, $2.5 \%$ yeast extract, $2.5 \% \mathrm{NaCl}, 0.1 \% \mathrm{~K}_{2} \mathrm{HPO}_{4}$, and $0.5 \%$ glucose $(\mathrm{w} / \mathrm{v})$ ]. When required for growth or selection, medium was supplemented with 5-Bromo-4-Chloro-3-Indolyl $\beta$-DGalactopyranoside (XGal) and/or the appropriate antibiotic at the following concentrations: erythromycin (Eri) $10 \mu \mathrm{g} \mathrm{ml}^{-1}$, ampicillin (Am) $100 \mu \mathrm{g} \mathrm{ml}^{-1}$, chloramphenicol (Clo) $10 \mu \mathrm{g} \mathrm{ml}^{-1}$ and $20 \mu \mathrm{g} \mathrm{ml}^{-1}$.

DNA manipulations. Plasmids were purified using NucleoSpin Plasmid kit (Macherey-Nagel) according to the protocol provided by the manufacturer. PCR fragments and enzymatic reactions were purified using 


\begin{tabular}{|c|c|c|}
\hline Plasmid & Characteristics & References \\
\hline pMAD & $\begin{array}{l}\text { E. coli-S. aureus shuttle vector with a thermosensitive origin of replication used for allelic } \\
\text { replacement }\end{array}$ & 45 \\
\hline pMAD::graRES & pMAD plasmid containing the allele for chromosomal restoration of graRS TCS & This study \\
\hline pMAD::saeRES & pMAD plasmid containing the allele for chromosomal restoration of saeRS TCS & This study \\
\hline pMAD:: $\Delta g r a \mathrm{X}$ & pMAD plasmid containing the allele for markerless deletion of graX gene & This study \\
\hline pMAD::graRES S(C227-A) & $\begin{array}{l}\text { pMAD plasmid containing the allele for chromosomal restoration of graRS S(C227-A) } \\
\text { isoform }\end{array}$ & This study \\
\hline pMAD::graRES S(C227-S) & pMAD plasmid containing the allele for chromosomal restoration of graRS S(C227-S) isoform & This study \\
\hline pMAD::graRES S(H129-Q) & $\begin{array}{l}\text { pMAD plasmid containing the allele for chromosomal restoration of graRS S(H120-Q) } \\
\text { isoform }\end{array}$ & This study \\
\hline pSA14 & $\begin{array}{l}\text { pM4 derivative carrying the promoterless E.coli lacZ gene for constructing transcriptional } \\
\text { fusions }\end{array}$ & 9 \\
\hline pSA14::mprFp & pSA14 containing the $m p r F$ promoter region & 9 \\
\hline pSA14::dltXp & pSA14 containing the $d l t \mathrm{X}$ promoter region & This study \\
\hline pSA14::sec4p & pSA14 containing the $\sec 4$ promoter region & This study \\
\hline
\end{tabular}

Table 2. Plasmid used in this study.

\begin{tabular}{|c|c|}
\hline Oligonucleotide & Sequence \\
\hline \multicolumn{2}{|l|}{ Restoration of TCS } \\
\hline gra-E & GGGCCATAAAAAGCCTCCAG \\
\hline gra-F & GTAGCTTCCGACTTGTGAGCC \\
\hline gra-A (EcoRI) & CCGGGAGCTCGAATTCCAAATAGATATTGCTGTATTCTTTATCGACCCAAC \\
\hline gra-D (BamHI) & GGGCGATATCGGATCCAACGCCACCTAAAACACTTTGTACAC \\
\hline G C227A Rv & CTAATAATCATACGGCACCATTTTATATC \\
\hline H C227A Fwd & GATATAAAATGGTGCCGTATGATTATTAG \\
\hline G C227S Rv & TCTAATAATCATACGAGACCATTTTATATC \\
\hline H C227S Fwd & AGATATAAAATGGTCTCGTATGATTATTAG \\
\hline G H129-Q Rv & GTTTTTATGTCTTGCACAAATTCTG \\
\hline H H129-Q Fwd & CAGAATTTGTGCAAGACATAAAAAC \\
\hline sae-E & AGTACAATTTGATGATGGTGTTGGTG \\
\hline sae-F & GATTTCACAGCACCCCTAGC \\
\hline sae-A (BamHI) & GGGCGATATCGGATCCCAAAAGGGTTATTTGAATGGATAGGC \\
\hline sae-D (NotI) & CCATGGCATGCATCGCTGTTCACATAACACTACAAATCGC \\
\hline graX A (BglII) & CACAGATCTGGTTGGTTATTGAGTGGTACATTTG \\
\hline graX B (XhoI) & CACCTCGAGCTAAAATACTCCTTTAAACTGTAACC \\
\hline graX C (XhoI) & CACCTCGAGGGTGATATGGATGCAAATAC \\
\hline $\operatorname{graX} D$ & GGAGGATCCTTTCGATTTGATTTTTTTTGGTAATAAG \\
\hline $\operatorname{graX} \mathrm{E}$ & GTTGTTATGCGATTCTGATACAAG \\
\hline graX F & TGTTTCGATTGCACTATCCATAC \\
\hline \multicolumn{2}{|l|}{ Reporter construction } \\
\hline pSA14-Fw & TGGAATTGTGAGCGGATAAC \\
\hline pSA14-Rv & CTCTTCGCTATTACGCCAG \\
\hline mprFp-Fw (PstI) & CTGCTGCAGTATAGATAACCATATTGTTC \\
\hline mprFp-Rv (BamHI) & GGAGGATCCTGATTCATTTTTTCACATCA \\
\hline dltXp-Fw (PstI) & GGCTGCAGGCGCTGATGATAATTCAATAA \\
\hline dltXp-Rv (BamHI) & CGGGATCCGATTTCATATTGCACCTCTTAAAG \\
\hline sec4p-Fw (PstI) & GGCTGCAGGAGTGTGAATATATAAACAATG \\
\hline sec4p-Rv (BamHI) & GCGGATCCTTATTCATTTTTATCTCCTTC \\
\hline mprFp-GFP-Fw (SalI) & GTCGTCGACGTATAGATAACCATATTGTTC \\
\hline mprFp-GFP-Rv (KpnI) & GGTGGTACCTGATTCATTTTTTCACATCA \\
\hline \multicolumn{2}{|c|}{ Complementation plasmid } \\
\hline graR Fw (KpnI) & GGGGTACCTCGAGAATGATATTGGGTGATATGG \\
\hline graR Rv (EcoRI) & GGGAATTCCAAATTATTCATGAGCCATATA \\
\hline
\end{tabular}

Table 3. Oligonucleotides used in this study. 
GeneJET Gel Extraction and DNA Cleanup Micro Kit (Thermo Scientific). FastDigest restriction enzymes, Rapid DNA ligation kit, Dreamtaq DNA polymerase and Phusion DNA polymerase were supplied by Thermo Scientific and used according to provided instructions. Sequence verification of PCR-amplified products and plasmid constructions was performed by Stab Vida. Transformation of Staphylococcus aureus was performed following previously standardized protocols ${ }^{43,44}$.

Allelic exchange of chromosomal genes. To generate markerless deletions, two fragments of at least $500 \mathrm{bp}$ that flanked upstream (primers A and B, Table 3) and downstream fragments (primers C and D, Table 3) of the region to be deleted were amplified by PCR. Amplified products were digested using the corresponding restriction enzymes (Table 3), purified and cloned by ligation into pMAD shuttle vector. To restore individual TCSs into $\triangle \mathrm{XV}$ chromosome, a fragment containing the two flanking regions used to generate the deletion ${ }^{15}$ and the original TCS sequence were amplified by PCR using chromosomal DNA from MW2 strain as template and oligonucleotide pair gra or sae 1-3. For restoration of the TCS with single amino acid substitutions at C227, graRS was amplified using MW2 chromosomal DNA as template and a two-step PCR protocol. First, the oligonucleotides A and $\mathrm{G}$ or $\mathrm{H}$ were used for generating two overlapping PCR products, while a second amplification step with A and D oligonucleotides using both purified PCR products as templates generated graRS S(C227-A) and graRS S(C227-S) isoforms. Such DNA fragments were purified, digested with the corresponding enzymes (see Table 3) and inserted by ligation into the pMAD shuttle vector ${ }^{45}$. Homologous recombination experiments were performed as previously described ${ }^{46}$. Final plasmidless erythromycin sensitive white colonies were tested by PCR using primers $\mathrm{E}$ and $\mathrm{F}$ (Table 3 ).

Reporter plasmid construction. pSA14 was used for the construction of different reporter plasmids. Promoter regions of $m p r F, d l t X$ and sec 4 were amplified by PCR using oligonucleotides described in Table 3. PCR fragments were purified and cloned into pSA14 through restriction enzymes to generate transcriptional fusion with lacZ. GraRS-dependent reporter plasmids were transformed via electroporation into S. aureus MW2, MW2 $\Delta$ graXRS, $\Delta \mathrm{X}, \Delta \mathrm{XV}$ Gra-RES and $\Delta \mathrm{XV}$ Gra-RES $\Delta$ graX, while plasmid harboring Sae-dependent sec4 promoter was inserted into MW2, $\triangle \mathrm{XV}$ and $\triangle \mathrm{XV}$ Sae-RES strains. To analyze $m p r F, d l t X$ and sec 4 expression, ON cultures were chemically lysed beta-galactosidase activity was measured.

High throughput beta-galactosidase-based screening. Screening of the 1280 off-patent FDAapproved drugs in Prestwick Chemical drug library (Prestwick Chemical) was based in a method for betagalactosidase assays in 96 well plates. Working solutions of the compounds were prepared in $50 \mu \mathrm{l}$ of sterile distilled-deionized water at a concentration of $20 \mu \mathrm{M}$, and combined with $50 \mu$ of a 1:30 dilution on $2 \times \mathrm{TSB}$ medium of an overnight $(\mathrm{ON})$ culture of $S$. aureus strains, thus generating $100 \mu \mathrm{l}$ of 1:60 cell dilution on $1 \times \mathrm{TSB}$ at a final concentration of $10 \mu \mathrm{M}$ of each drug. Plates were incubated during $24 \mathrm{~h}$ at $37^{\circ} \mathrm{C}$ and, upon incubation, $\mathrm{OD}_{600 \mathrm{~nm}}$ was measured (Multiskan Go; Thermo Scientific). Bacterial cells were subsequently lysed by the addition of $100 \mu \mathrm{l}$ well ${ }^{-1}$ of $\mathrm{Z}$ buffer supplemented with lysostaphin $\left(0.5 \mathrm{mg} \mathrm{ml}^{-1}\right)$ during $2 \mathrm{~h}$ at $37^{\circ} \mathrm{C}$; Next, $30 \mu \mathrm{l}$ well ${ }^{-1}$ of Ortho-Nitrophenyl-beta-galactoside (ONPG, $4 \mathrm{mg} \mathrm{ml}^{-1}$ ) was added and, when required, the reaction was stopped with $100 \mu$ well $^{-1}$ of $1 \mathrm{M} \mathrm{Na}_{2} \mathrm{CO} 3$. $\mathrm{OD}_{420}$ and $\mathrm{OD}_{550}$ values were finally recorded for Miller Units calculation. Untreated reporter $\triangle \mathrm{XV}$-GraRES and $\triangle \mathrm{XV}$ strains were included in every plate as internal controls. Experiments were carried out in triplicate.

Phagocytosis and killing of S. aureus by human PMNs. Phagocytosis and killing of S. aureus by human neutrophils in presence of selected compounds was determined as described before ${ }^{47}$. Polymorphonuclear cells (PMNs) were isolated from healthy human heparinized-defibrinated blood (Seralab Logistics) using Ficoll-Plaque PREMIUM (GE-Healthcare) according to manufacturer's protocol and resuspended at a final concentration of $1 \times 10^{7} \mathrm{PMNs} \mathrm{ml}^{-1}$ in HBSS supplemented with human serum. S. aureus strains were cultured to the early stationary phase and $10 \mathrm{ml}$ of culture were centrifuged, washed twice with sterile PBS and resuspended in Hank's Balanced Salt Solution (HBSS) supplemented with human serum at a final concentration of $4 \times 10^{5}$ bacteria $\mathrm{ml}^{-1}$. Finally, $0.2 \mathrm{ml}$ of PMNs solution was mixed with $0.2 \mathrm{ml}$ of $S$. aureus solution and $600 \mu \mathrm{l}$ of HBSS supplemented with human serum. Compounds were added at a final concentration of $5 \mu \mathrm{M}$. After incubation at $37^{\circ} \mathrm{C}$ for $30 \mathrm{~min}$, each sample was treated with gentamicin $100 \mathrm{mg} \mathrm{ml}^{-1}$ and then $100 \mu \mathrm{l}$ of each mixture were added to $1 \mathrm{ml}$ of $\mathrm{pH} 11$ solution. Finally, serial dilutions were plated on TSA to determine the number of colonyforming units (CFU) in presence of the different compounds. All data were referred to initial CFU number.

Mouse infection model. The experimental animal study was reviewed and approved by the "Comité de Ética, Experimentación Animal y Bioseguridad" of the Universidad de Navarra-Centro de Investigación Médica Aplicada (CIMA). Work was carried out at the CIMA animal facility under the principles and guidelines described in the "European Directive 86/609/EEC" for the protection of animals used for experimental purposes. Six-week-old female Swiss mice (20-25 g) were obtained from ENVIGO and confined in groups of 6 animals.

The model was performed as previously described ${ }^{50}$. Briefly, $10 \mathrm{~cm}$ fragments of commercial brailed silk (TC-15, Lorca Marín) were contaminated with $4 \times 10^{6} \mathrm{CFU} \mathrm{cm} \mathrm{cm}^{-1}$ of $S$. aureus MW2 or S. aureus MW2 $\Delta$ graXRS strains by immersion during $30 \mathrm{~min}$. Fragments were then blotted-dried. One day prior to the experiment, the interscapular skin was shaved using a sharp razor. On the day of the infection, superficial wounds were produced on the exposed back surface though a longitudinal midline incision of $2 \mathrm{~cm}$ approximately. The skin of either side of the incision was retracted, and the wound was infected by stitching it with contaminated suture and a suturing needle. Wounds were topically treated $1 \mathrm{~h}$ and $8 \mathrm{~h}$ after infection with approximately $100 \mu \mathrm{l}$ of hydrogel 
formulations containing 0.125 and $2.5 \mathrm{mg} / 100 \mu \mathrm{l}$. The hydrogel base without any active substance was applied in the control group. Treatments were repeated $24 \mathrm{~h}$ after infection and mice were sacrificed $24 \mathrm{~h}$ after the last application. Finally, the wounded tissue was resected, homogenized in PBS, and dilution series of homogenates was plated on TSB agar for enumeration of CFU (output). After an overnight incubation at $37^{\circ} \mathrm{C}$, CFU gr of tissue ${ }^{-1}$ were calculated and expressed as $\log _{10}$.

Statistical analysis. Data generated by PMN-mediated killing assay were compared using ANOVA, applying Tukey's pairwise as post hoc test. Data obtained from the bacterial counts in the murine model were treated and compared using Kruskal-Wallis test, Mann-Whitney pairwise and Dunn's post hoc tests. All tests were two-sided, and the significance level was 5\%. The statistical analysis was performed with Past and R softwares.

Received: 4 December 2019; Accepted: 21 September 2020

Published online: 21 October 2020

\section{References}

1. Kelly, R. \& Davies, S. C. Tackling antimicrobial resistance globally. Med. J. Aust. 207, 371-373 (2017).

2. Rasigade, J.-P., Dumitrescu, O. \& Lina, G. New epidemiology of Staphylococcus aureus infections. Clin. Microbiol. Infect. 20, 587-588 (2014).

3. Wertheim, H. F. et al. The role of nasal carriage in Staphylococcus aureus infections. Lancet Infect. Dis. 5, 751-762 (2005).

4. Balasubramanian, D., Harper, L., Shopsin, B. \& Torres, V. J. Staphylococcus aureus pathogenesis in diverse host environments. Pathog. Dis. 75, ftx005 (2017).

5. Stock, A. M., Robinson, V. L. \& Goudreau, P. N. Two-component signal transduction. Annu. Rev. Biochem. 69, 183-215 (2000).

6. Capra, E. J. \& Laub, M. T. Evolution of two-component signal transduction systems. Annu. Rev. Microbiol. 66, 325-347 (2012).

7. Kawada-Matsuo, M., Yoshida, Y., Nakamura, N. \& Komatsuzawa, H. Role of two-component systems in the resistance of Staphylococcus aureus to antibacterial agents. Virulence 2, 427-430 (2011).

8. Boyle-Vavra, S., Yin, S., Jo, D. S., Montgomery, C. P. \& Daum, R. S. VraT/YvqF is required for methicillin resistance and activation of the VraSR regulon in Staphylococcus aureus. Agencia Eur. Med. 57, 83-95 (2013).

9. Falord, M., Mäder, U., Hiron, A., Débarbouillé, M. \& Msadek, T. Investigation of the Staphylococcus aureus GraSR regulon reveals novel links to virulence, stress response and cell wall signal transduction pathways. PLoS ONE 6, e21323 (2011).

10. Haag, A. F. \& Bagnoli, F. The role of two-component signal transduction systems in Staphylococcus aureus virulence regulation. Life Sci. J. 6, 145-198 (2015).

11. Bem, A. E. et al. Bacterial histidine kinases as novel antibacterial drug targets. ACS Chem. Biol. 10, 213-224 (2015).

12. Okada, A. et al. Walkmycin B targets WalK (YycG), a histidine kinase essential for bacterial cell growth. J. Antibiot. (Tokyo) 63, 89-94 (2010).

13. Hilliard, J. J., Goldschmidt, R. M., Licata, L., Baum, E. Z. \& Bush, K. Multiple mechanisms of action for inhibitors of histidine protein kinases from bacterial two-component systems. Antimicrob. Agents Chemother. 43, 1693-1699 (1999).

14. Gotoh, Y. et al. Two-component signal transduction as potential drug targets in pathogenic bacteria. Curr. Opin. Microbiol. 13, 232-239 (2010).

15. Villanueva, M. et al. Sensory deprivation in Staphylococcus aureus. Nat. Commun. 9, 523 (2018).

16. Kraus, D. et al. The GraRS regulatory system controls Staphylococcus aureus susceptibility to antimicrobial host defenses. BMC Microbiol. 8, 85 (2008).

17. Yang, S.-J. et al. The Staphylococcus aureus two-component regulatory system, GraRS, senses and confers resistance to selected cationic antimicrobial peptides. Infect. Immun. 80, 74-81 (2012).

18. Cheung, A. L. et al. Site-specific mutation of the sensor kinase GraS in Staphylococcus aureus alters the adaptive response to distinct cationic antimicrobial peptides. Infect. Immun. 82, 5336-5345 (2014).

19. Chaili, S. et al. The GraS sensor in Staphylococcus aureus mediates resistance to host defense peptides differing in mechanisms of action. Infect. Immun. 84, 459-466 (2016).

20. Flannagan, R. S., Kuiack, R. C., McGavin, M. J. \& Heinrichs, D. E. Staphylococcus aureus uses the GraXRS regulatory system to sense and adapt to the acidified phagolysosome in macrophages. MBio 9, 561 (2018).

21. Muzamal, U., Gomez, D., Kapadia, F. \& Golemi-Kotra, D. Diversity of two-component systems: insights into the signal transduction mechanism by the Staphylococcus aureus two-component system GraSR. F1000Res. 3, 252 (2014).

22. Liu, Q., Yeo, W.-S. \& Bae, T. The SaeRS two-component system of Staphylococcus aureus. Genes (Basel) 7, 81 (2016).

23. Eales, K. L., Wilkinson, E. A., Cruickshank, G., Tucker, J. H. R. \& Tennant, D. A. Verteporfin selectively kills hypoxic glioma cells through iron-binding and increased production of reactive oxygen species. Sci. Rep. 8, 14358 (2018).

24. Shimizu, T., Lengalova, A., Martínek, V. \& Martínková, M. Heme: emergent roles of heme in signal transduction, functional regulation and as catalytic centres. Chem. Soc. Rev. 48, 5624-5657 (2019).

25. van der Meer, J. W. M., Fears, R., Davies, D. S. C. \& ter Meulen, V. Antimicrobial innovation: combining commitment, creativity and coherence. Nat. Rev. Drug Discov. 13, 709-710 (2014).

26. Kong, C., Neoh, H. \& Nathan, S. Targeting Staphylococcus aureus toxins: a potential form of anti-virulence therapy. Toxins (Basel) 8, 72 (2016).

27. Thangamani, S., Mohammad, H., Abushahba, M. F. N., Sobreira, T. J. P. \& Seleem, M. N. Repurposing auranofin for the treatment of cutaneous staphylococcal infections. Int. J. Antimicrob. Agents 47, 195-201 (2016).

28. Yeo, W.-S. et al. The FDA-approved anti-cancer drugs, streptozotocin and floxuridine, reduce the virulence of Staphylococcus aureus. Sci. Rep. 8, 2521 (2018)

29. Thangamani, S., Mohammad, H., Younis, W. \& Seleem, M. Drug repurposing for the treatment of staphylococcal infections. Curr. Pharm. Des. 21, 2089-2100 (2015).

30. Li, M. et al. The antimicrobial peptide-sensing system aps of Staphylococcus aureus. Mol. Microbiol. 66, 1136-1147 (2007).

31. Falord, M., Karimova, G., Hiron, A. \& Msadek, T. GraXSR proteins interact with the VraFG ABC transporter to form a fivecomponent system required for cationic antimicrobial peptide sensing and resistance in Staphylococcus aureus. Antimicrob. Agents Chemother. 56, 1047-1058 (2012).

32. Miró-Canturri, A., Ayerbe-Algaba, R. \& Smani, Y. Drug repurposing for the treatment of bacterial and fungal infections. Front. Microbiol. 10, 41 (2019).

33. Gautret, P. et al. Hydroxychloroquine and azithromycin as a treatment of COVID-19: results of an open-label non-randomized clinical trial. Int. J. Antimicrob. Agents 56, 105949 (2020). 
34. Khodaverdian, V. et al. Discovery of antivirulence agents against methicillin-resistant Staphylococcus aureus. Antimicrob. Agents Chemother. 57, 3645-3652 (2013).

35. Hendrix, A. S. et al. Repurposing the nonsteroidal anti-inflammatory drug diflunisal as an osteoprotective, antivirulence therapy for Staphylococcusaureus osteomyelitis. Antimicrob. Agents Chemother. 60, 5322-5330 (2016).

36. Loi, V. V. et al. Redox-sensing under hypochlorite stress and infection conditions by the Rrf2-family repressor HypR in Staphylococcus aureus. Antioxid. Redox Signal. 29, 615-636 (2018).

37. Loi, V. V. et al. Staphylococcus aureus responds to allicin by global S-thioallylation-role of the Brx/BSH/YpdA pathway and the disulfide reductase MerA to overcome allicin stress. Free Radic. Biol. Med. 139, 55-69 (2019).

38. Rapun-Araiz, B. et al. Systematic reconstruction of the complete two-component sensorial network in Staphylococcus aureus. mSystems 5, e00511-e00520. https://doi.org/10.1128/mSystems.00511-20 (2020).

39. Malpica, R., Franco, B., Rodriguez, C., Kwon, O. \& Georgellis, D. Identification of a quinone-sensitive redox switch in the ArcB sensor kinase. Proc. Natl. Acad. Sci. 101, 13318-13323 (2004).

40. Fridman, M. et al. Two unique phosphorylation-driven signaling pathways crosstalk in Staphylococcus aureus to modulate the cell-wall charge: Stk1/Stp1 meets GraSR. Biochemistry 52, 7975-7986 (2013).

41. AlAmri, M. A., Kadri, H., Alderwick, L. J., Jeeves, M. \& Mehellou, Y. The photosensitising clinical agent verteporfin is an inhibitor of SPAK and OSR1 kinases. ChemBioChem 19, 2072-2080 (2018).

42. Elyasi, S., Khalili, H., Dashti-Khavidaki, S. \& Mohammadpour, A. Vancomycin-induced nephrotoxicity: mechanism, incidence, risk factors and special populations. A literature review. Eur. J. Clin. Pharmacol. 68, 1243-1255 (2012).

43. Lee, J. C. Electrotransformation of Staphylococci. In Electroporation Protocols for Microorganisms (ed. Nickoloff, J. A.) 209-216 (Humana Press, Totowa, 1995). https://doi.org/10.1385/0-89603-310-4:209.

44. Schenk, S. \& Laddaga, R. A. Improved method for electroporation of Staphylococcus aureus. FEMS Microbiol. Lett. 94, 133-138 (1992).

45. Arnaud, M., Chastanet, A. \& Debarbouille, M. New vector for efficient allelic replacement in naturally nontransformable, lowGC-content, Gram-positive bacteria. Appl. Environ. Microbiol. 70, 6887-6891 (2004).

46. Valle, J. et al. SarA and not $\sigma B$ is essential for biofilm development by Staphylococcus aureus. Mol. Microbiol. 48, 1075-1087 (2003).

47. Peschel, A. et al. Staphylococcus aureus resistance to human defensins and evasion of neutrophil killing via the novel virulence factor Mprf is based on modification of membrane lipids with L-lysine. J. Exp. Med. 193, 1067-1076 (2001).

48. Novick, R. P. Molecular Biology of the Staphylococci. VCH Publ. 1-37 (1990).

49. Baba, T. et al. Genome and virulence determinants of high virulence community-acquired MRSA. Lancet 359, 1819-1827 (2002).

50. McRipley, R. J. \& Whitney, R. R. Characterization and quantitation of experimental surgical-wound infections used to evaluate topical antibacterial agents. Antimicrob. Agents Chemother. 10, 38-44 (1976).

\title{
Acknowledgements
}

This study was supported by Centre for the Development of Industrial Technology (CDTI), (NEO16RECOMBINA; EXP 00112635/SNEO-20161233) Work in the Laboratory of Microbial Pathogenesis is funded by the Spanish Ministry of Science, Innovation and Universities grant BIO2017-83035-R (AEI/FEDER, EU).

\section{Author contributions}

C.L., I.L. and J.-P. conceived and designed the experiments, J.-P. performed the experiments. C.G. and B.R. performed the murine model. C.L. analyzed the data and wrote the first draft of the manuscript. I.L. and J.-P. made significant contributions to consecutive versions of the manuscript. All authors reviewed the manuscript and approved its final version.

\section{Additional information}

Supplementary information is available for this paper at https://doi.org/10.1038/s41598-020-74873-5.

Correspondence and requests for materials should be addressed to C.L.

Reprints and permissions information is available at www.nature.com/reprints.

Publisher's note Springer Nature remains neutral with regard to jurisdictional claims in published maps and institutional affiliations.

\begin{abstract}
Open Access This article is licensed under a Creative Commons Attribution 4.0 International License, which permits use, sharing, adaptation, distribution and reproduction in any medium or format, as long as you give appropriate credit to the original author(s) and the source, provide a link to the Creative Commons licence, and indicate if changes were made. The images or other third party material in this article are included in the article's Creative Commons licence, unless indicated otherwise in a credit line to the material. If material is not included in the article's Creative Commons licence and your intended use is not permitted by statutory regulation or exceeds the permitted use, you will need to obtain permission directly from the copyright holder. To view a copy of this licence, visit http://creativecommons.org/licenses/by/4.0/.
\end{abstract}

(c) The Author(s) 2020 